\title{
Effect of hospital and surgeon volume on postoperative outcomes after distal gastrectomy for gastric cancer based on data from 145,523 Japanese patients collected from a nationwide web-based data entry system
}

\author{
Masaaki Iwatsuki ${ }^{1} \cdot$ Hiroyuki Yamamoto ${ }^{2,3} \cdot$ Hiroaki Miyata ${ }^{2,3} \cdot$ Yoshihiro Kakeji $^{4} \cdot$ Kazuhiro Yoshida $^{5}$. \\ Hiroyuki Konno ${ }^{6} \cdot$ Yasuyuki Seto $^{7} \cdot$ Hideo Baba $^{1}$ (]
}

Received: 19 July 2018 / Accepted: 29 September 2018 / Published online: 9 October 2018

(c) The International Gastric Cancer Association and The Japanese Gastric Cancer Association 2018

\begin{abstract}
Background Despite interest in surgeon and hospital volume effects on distal gastrectomy, clinical significance has not been confirmed in a large-scale population. We studied to clarify the effects of surgeon and hospital volume on postoperative mortality after distal gastrectomy for gastric cancer among Japanese patients in a nationwide web-based data entry system. Methods We extracted data on distal gastrectomy for gastric cancer from the National Clinical Database between 2011 and 2015. The primary outcome was operative mortality. Hospital volume was divided into 3 tertiles: low (1-22 cases per year), medium (23-51) and high (52-404). Surgeon volume was divided into the 5 groups: 0-3, 4-10, 11-20, 21-50, 51+ cases per year. We calculated the $95 \%$ confidence interval (CI) for the mortality rate based on odds ratios (ORs) estimated from a hierarchical logistic regression model.

Results We analyzed 145,523 patients at 2182 institutions. Operative mortality was $1.9 \%$ in low-, $1.0 \%$ in medium- and $0.5 \%$ in high-volume hospitals. The operative mortality rate decreased definitively with surgeon volume, $1.6 \%$ in the $0-3$ group and $0.3 \%$ in the $51+$ group. After risk adjustment for surgeon and hospital volume and patient characteristics, hospital volume was significantly associated with operative morality (medium: OR $0.64,95 \% \mathrm{CI} 0.56-0.73, P<0.001$; high: OR $0.42,95 \%$ CI $0.35-0.51, P<0.001)$.

Conclusions We demonstrate that hospital volume can have a crucial impact on postoperative mortality after distal gastrectomy compared with surgeon volume in a nationwide population study. These findings suggest that centralization may improve outcomes after distal gastrectomy.
\end{abstract}

Keywords Gastric cancer · Distal gastrectomy · Hospital volume

Hideo Baba

hdobaba@kumamoto-u.ac.jp

1 Department of Gastroenterological Surgery, Graduate School of Medical Sciences, Kumamoto University, 1-1-1 Honjo, Chuo-ku, Kumamoto 860-8556, Japan

2 Department of Healthcare Quality Assessment, Graduate School of Medicine, The University of Tokyo, Tokyo, Japan

3 Department of Health Policy and Management, School of Medicine, Keio University, Tokyo, Japan
4 Database Committee, The Japanese Society of Gastroenterological Surgery, Tokyo, Japan

5 Department of Surgical Oncology, Gifu University School of Medicine, Gifu, Japan

6 Hamamatsu University School of Medicine, Hamamatsu, Japan

7 The Japanese Society of Gastroenterological Surgery, Tokyo, Japan 


\section{Introduction}

Gastric cancer is a common malignancy and is associated with a high mortality rate worldwide [1]. Notably, gastric cancer is the second leading cause of cancer-related death, with the highest mortality rates in East Asia, including Japan, Korea and China. Despite the recent progress in cancer treatment, the prognosis of patients with advanced gastric cancer remains poor. Gastrectomy with regional lymph node dissection is the most effective treatment for gastric cancer. However, postoperative complications can lead to adverse effects, not only on the overall survival, but also on the disease-free survival of patients with gastric cancer, treated with curative intent [2, 3]. In particular, inflammatory postoperative complications may have a significant negative impact on the prognosis of patients with gastric cancer [4].

For advanced gastric cancer, gastrectomy with D2 lymph node dissection is recommended worldwide [5-7]. However, the European Society for Medical Oncology (ESMO) guideline notes that, in Western countries, medically fit patients should undergo D2 lymph node dissection that is carried out in specialized, high-volume centers with appropriate surgical expertise and postoperative care [7], because hospitals performing larger numbers of esophagogastric cancer resections had a lower 30-day mortality rate across Europe [8]. In general, postoperative complications are reported to be associated with hospital volume in several types of disease [9]. Recently, investigations of not only hospital volume, but also surgeon volume, have shed light on surgery for gastrointestinal cancer, emergency surgery and other surgeries [10-13]. With regard to gastric cancer, Coupland et al. recently reported that increasing hospital volume was associated with lower mortality, suggesting that further centralization of esophageal and gastric cancer surgical services was warranted in England [14]. Recently, investigation of the relationship between surgeon volume and mortality has received attention similar to that for hospital volume. Although some reports suggested that the minimum surgeon volume for gastrectomy was approximately 10-15 gastrectomies per year [12, 15], further evaluation is required in a large-scale cohort.

In Japan, the National Clinical Database (NCD) was founded in 2010 as the parent body of the database system linked to the board certification system [16]. The NCD project, which commenced recordkeeping in January 2011, contains records of $\geq 95 \%$ of the surgeries performed by regular surgeons in Japan. Almost 5000 facilities have enrolled, and over 9,100,000 cases have been registered as of the end of December 2016. Risk stratification studies based on data from the NCD database have been reported for gastrectomy for Japanese patients with gastric cancer
$[17,18]$. These risk scoring systems use a risk calculator available on the NCD Web site (http://www.ncd.or.jp/) for physicians in clinical practice to inform patients and their families of the risk associated with gastrectomy [19].

In this study, to clarify the impact of surgeon and hospital volume on postoperative mortality for distal gastrectomy, we evaluated data from 145,523 Japanese patients with gastric cancer enrolled in the nationwide web-based data entry system.

\section{Methods}

\section{Data collection}

From 2011, the NCD collected data on more than 9,100,000 surgical cases from approximately 5000 hospitals. In the gastroenterological surgery section, the database registered all surgical cases that fell into this category; in addition, it required detailed input items for 8 procedures, including gastrectomy, that were determined to represent the performance of surgery in each specialty. The NCD constructed software for an internet-based data collection system, and the data managers of the participating hospitals were responsible for forwarding their data to the NCD office. The NCD ensures traceability of its data by maintaining continuity in the staff who approve the data, the staff of the departments in charge of annual cases, and the data-entry personnel. It also validates data consistency via random inspections of participating institutions.

In this study, we focused on the specific NCD section for gastrointestinal surgery. Briefly, potential independent variables included patient demographics, pre-existing comorbidities, preoperative laboratory values, and operative data.

\section{Patients}

A total of 145,523 patients who underwent distal gastrectomy for gastric cancer at 2182 institutions between January 1, 2011 and December 31, 2015, were eligible for analysis. Sixty-three records with missing data on patient age, sex, or outcome were excluded.

\section{Endpoint}

The primary outcome measure of this study was 30-day and operative mortalities. Operative mortality was defined as death during the index hospitalization, regardless of the length of hospital stay ( $\leq 90$ days), as well as after hospital discharge within 30 days from the operation date. 


\section{Hospital and surgeon volume}

We divided hospital volume in the previous year into the following 3 tertiles: category low (1-22 cases per year), category medium (23-51 cases) and category high (52-404). We defined surgeon volume as the number of gastrectomies performed by a patient's surgeon in the previous year. Surgeon volume was divided into the following five groups: $0-3,4-10,11-20,21-50,51+$ cases per year.

\section{Statistical methods}

All statistical calculations were performed with STATA 15 (STATA Corp., TX, USA). We compared median values with the Kruskal-Wallis test for operation time and estimated blood loss, and chi-squared test for all other variables. All $P$ values were two-sided, and we considered $P<0.05$ as statistically significant. First, we analyzed the relationship between annual surgical volume (hospital volume or surgeon volume) and surgical mortality after gastrectomy with hierarchical logistic regression models accounting for clustering of patients by surgeons and hospital levels. To adjust for patient-level risk factors, the following variables, which were used in the scoring system established by NCD data [18], were utilized: demographic factors, such as age category and sex; preoperative functional status, such as need for total assistance with activities of daily living (ADL); history of cerebrovascular disease; weight loss more than 10\%; uncontrolled ascites; ASA score class 3 or more; pre-existing comorbidities, such as the presence of respiratory distress, disseminated cancer, chronic corticosteroid use; operative factors, such as emergency surgery and laparoscopic gastrectomy; and preoperative laboratory data, such as white blood cell count more than $11,000 / \mu \mathrm{L}$, anemia (hemoglobin: males, $<13.5 \mathrm{~g} / \mathrm{dL}$; females, $<12.5 \mathrm{~g} / \mathrm{dL}$; or hematocrit: males $<37 \%$; females $<32 \%$ ), serum albumin less than $3.8 \mathrm{~g} /$ $\mathrm{dL}$, alkaline phosphatase more than $340 \mathrm{IU} / \mathrm{L}$, serum creatinine more than $1.2 \mathrm{mg} / \mathrm{dL}$, serum Na less than $135 \mathrm{mEq} / \mathrm{L}$, and prothrombin time-international normalized ratio more than 1.1 , low platelet count $\left(<12 \times 10^{4} / \mu \mathrm{L}\right)$, aspartate aminotransferase more than $40 \mathrm{IU} / \mathrm{L}$, increased level of total bilirubin $(>2 \mathrm{mg} / \mathrm{dL})$, and activated partial thromboplastin time more than $40 \mathrm{~s}$. In addition, to illustrate the relationship between operative mortality and surgeon volume as a continuous variable, generalized estimating equation logistic regression models were utilized, in which a restricted cubic spline model was implemented (Fig. 2). All procedures were conducted in accordance with the ethical standards of the respective committees on human experimentation (Institutional and National) and with the Helsinki Declaration. An ethics committee that includes members of the Japanese Surgical Society ethics board, lawyers, patient representatives and experts on information security that considered the ethical propriety of the entire initiative approved it and made the review process public on the Japan Surgical Society website. The use of data from the registry for retrospective observational studies was approved by The Japanese Society of Gastroenterological Surgery committee and the institutional Review Board of Kumamoto University committee, and individual written or verbal informed consent was waived because of the retrospective design.

\section{Results}

We retrieved data on a total of 145,523 patients who underwent distal gastrectomy for gastric cancer by 11,914 surgeons at 2182 institutions from January 2011 to December 2015. Hospital volume ranged from 1 to 404 gastrectomies per year. Annual hospital volume was distributed among the tertiles as follows: category low ( $n=49,161 ; 1-22$ cases), medium ( $n=46,729 ; 23-51$ cases $)$ and high ( $n=47,633$; 52-404 cases). Patient demographic data and preoperative risk assessment according to hospital volume category are summarized in Table 1. Low-volume hospitals had significantly older patients and poorer-risk patients with various comorbidities and organ dysfunctions. Table 1 shows the surgical outcomes according to hospital volume category. More laparoscopic gastrectomies were performed in the high-volume hospitals $(P<0.001)$. Significantly shorter operation time and less estimated blood loss were observed in the high-volume hospitals $(P<0.001)$. Operative mortality was $1.9 \%$ in low-volume hospitals, $1.0 \%$ in medium and $0.5 \%$ in high. A significant reduction in mortality rate was observed according to hospital volume $(P<0.001)$. Regarding surgical complications, anastomotic leakage was more frequently observed in low-volume hospitals, but the incidence of pancreatic fistula was higher in high-volume hospitals $(P<0.001)$. The rate of all nonsurgical complications including pneumonia, the reoperation rate and the rate of septic shock were significantly higher in low-volume hospitals $(P<0.001)$.

Patient demographic data and preoperative risk assessment according to surgeon volume are summarized in Table 2. Low-volume surgeons had operated on significantly older patients and poorer-risk patients with various comorbidities and organ dysfunctions, similar to the situation with low-volume hospitals. Table 2 shows the surgical outcomes according to surgeon volume category. More laparoscopic gastrectomies were performed by the high-volume surgeons $(P<0.001)$. Significantly shorter operation time and less estimated blood loss were observed among the high-volume surgeons $(P<0.001)$. The operative mortality rate decreased definitively with surgeon volume, $1.6 \%$ in the $0-3$ group and $0.3 \%$ in the $51+$ group. Regarding surgical complications, anastomotic leakage was more frequently observed among 


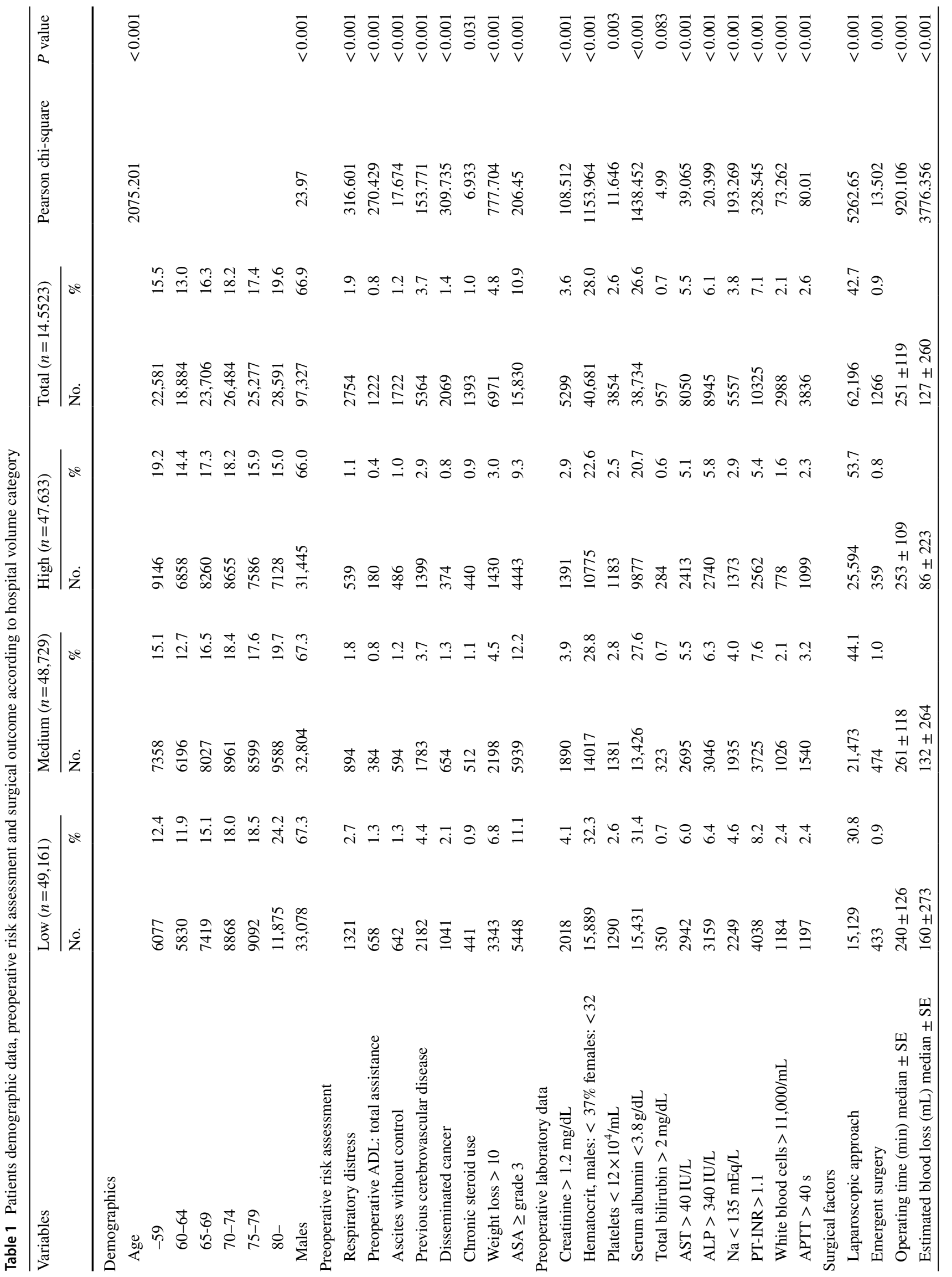




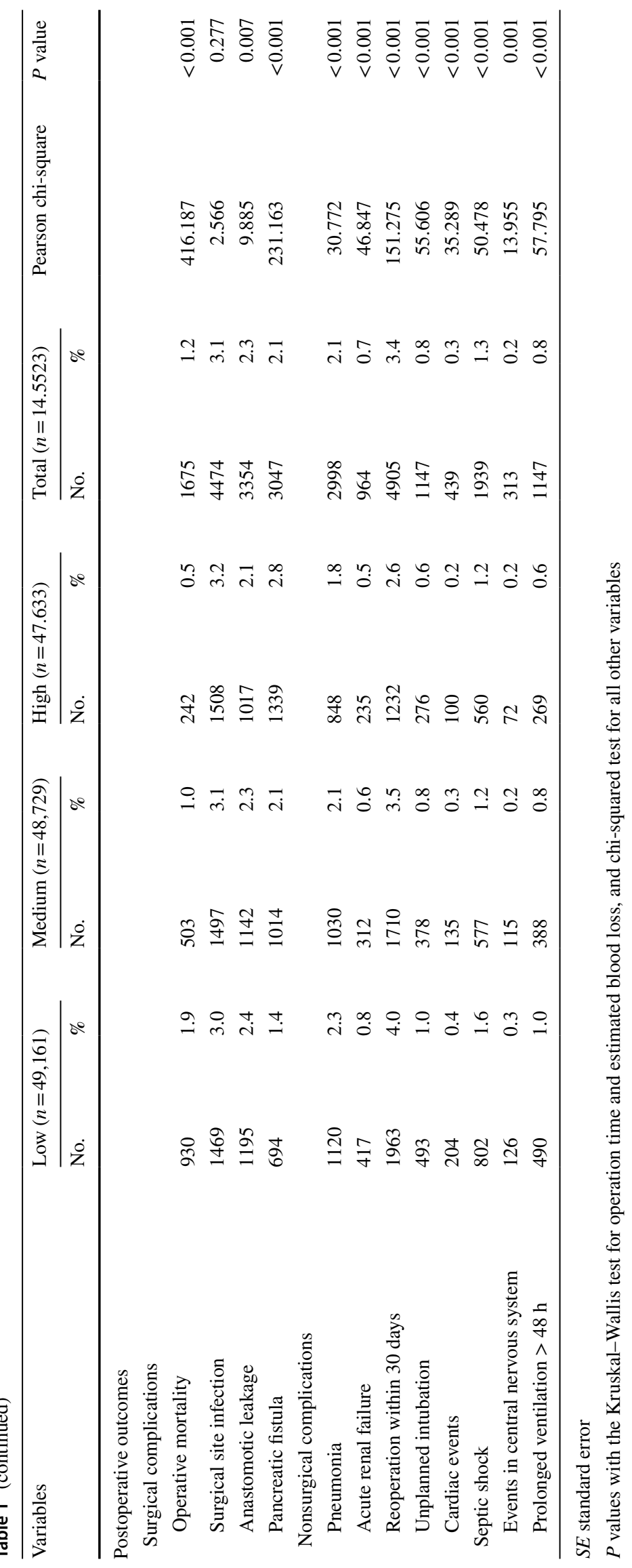




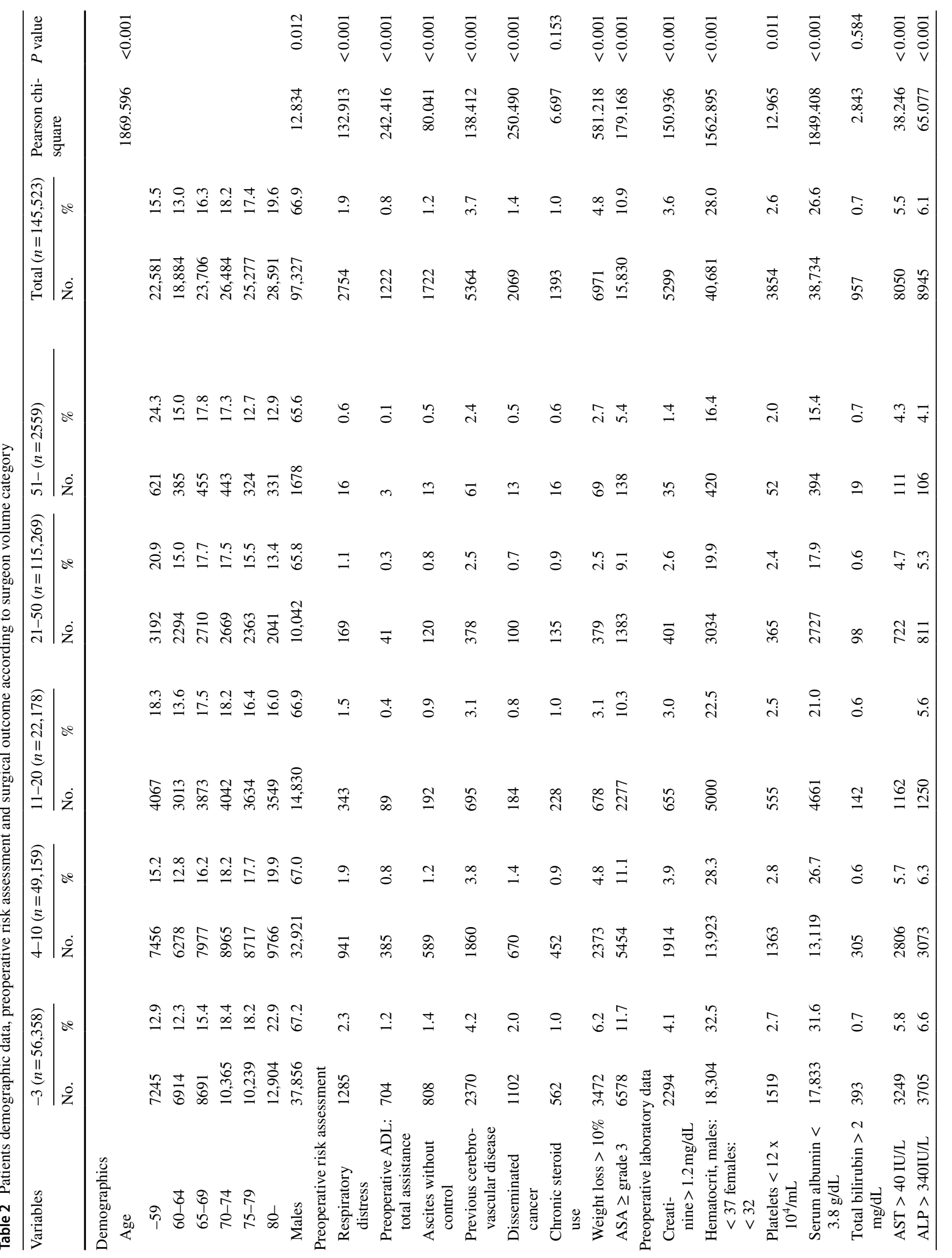




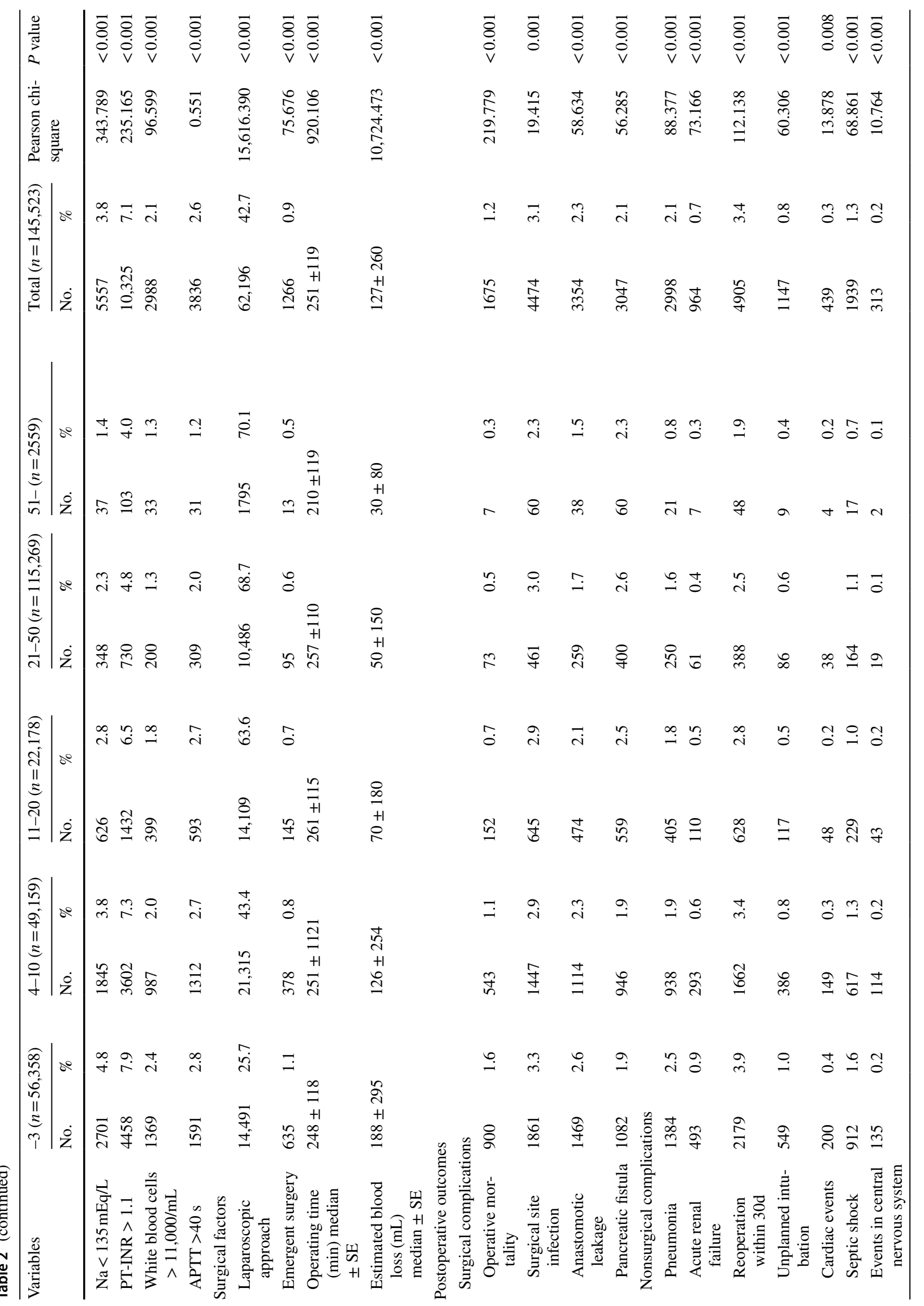


low-volume surgeons, but the incidence of pancreatic fistula was higher among high-volume surgeons $(P<0.001)$. The rate of all nonsurgical complications including pneumonia, the reoperation rate and the rate of septic shock were significantly higher among low-volume surgeons $(P<0.001)$.

Figure 1 summarizes the $95 \%$ CIs for overall mortality after distal gastrectomy from the hierarchical logistic regression models. The lowest volume surgeons ( -3 cases/year) were significantly associated with higher mortality (Fig. 1a OR, 1.32, 95\% CI, 1.09-1.61, $P<0.001$ ), and higher-volume hospitals were significantly associated with a decreased risk of mortality in a dose-dependent manner (Fig. 1b category 2: OR $0.63,95 \%$ CI $0.55-0.72 ; P<0.001$, category 3 : OR, $0.41,95 \%$ CI $0.34-0.49, P<0.001)$. After risk adjustment for surgeon and hospital volume, hospital volume was significantly associated with operative morality, whereas surgeon volume was not (Fig. 1c, d category 2: OR 0.64, $95 \%$ CI $0.56-0.73, P<0.001$; category 3 : OR $0.42,95 \%$ CI $0.35-0.51, P<0.001)$.

Furthermore, the OR for operative mortality gradually decreased in a surgeon volume-dependent manner after risk adjustment for patient-level factors such as demographic factors, preoperative functional status, pre-existing comorbidities, operative factors and preoperative laboratory data (Fig. 2a) and adding hospital volume (Fig. 2b).

\section{Discussion}

In this nationwide study, we found that both hospital and surgeon volume were associated with postoperative mortality after gastrectomy among 145,523 Japanese patients with gastric cancer. In particular, hospital volume had a strong influence on mortality, which is comparable to previously reported findings $[8,14,20,21]$. However, to the best of our knowledge, this is the largest study to show a correlation between hospital and surgeon volume and mortality after gastric cancer surgery. The annual surgeon volume can be a proxy for medical care quality [22], however, we need to pay considerable attention to this evaluation, because annual surgeon volume alone may not cover the underlying issues completely, such as hospital volume, specialization, and mentorship opportunities [13, 23].

Several previous studies have demonstrated an influence of surgeon volume on postoperative outcomes among patients undergoing elective and emergent surgery [13, 24]. With regard to gastric cancer surgery, patients treated by high-volume and experienced surgeons have definitively better short- and long-term outcomes [25, 26]. Our data shows that both hospital and surgeon volume were associated with morbidity and mortality after gastrectomy. However, after risk adjustment, higher-volume hospitals were significantly associated with a decreased risk of mortality 
a

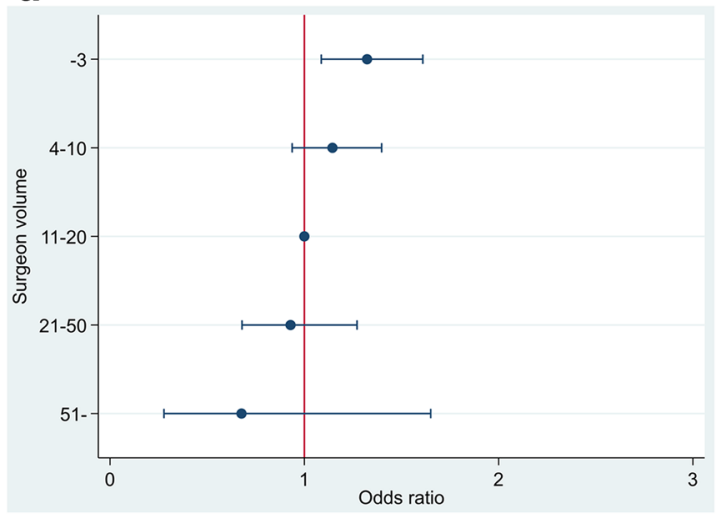

b

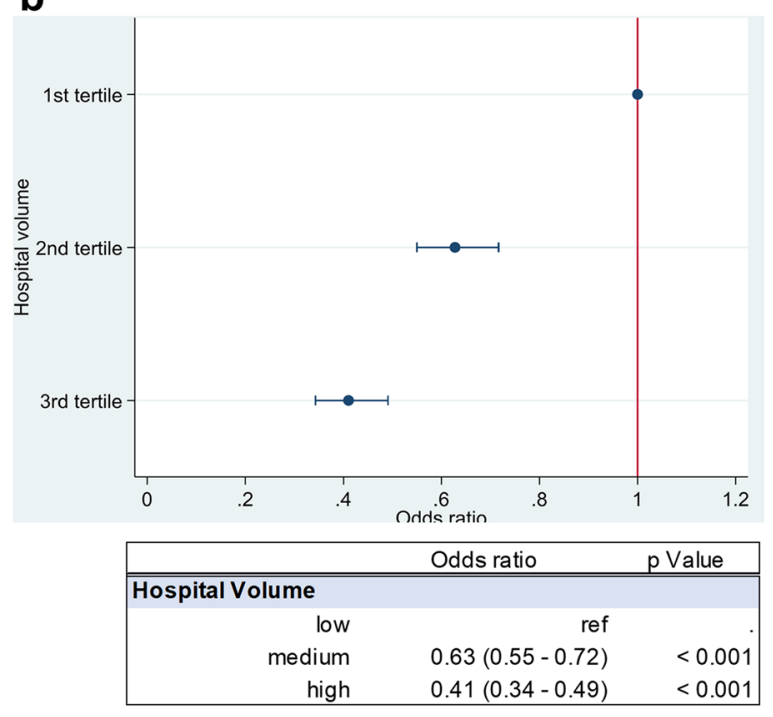

C

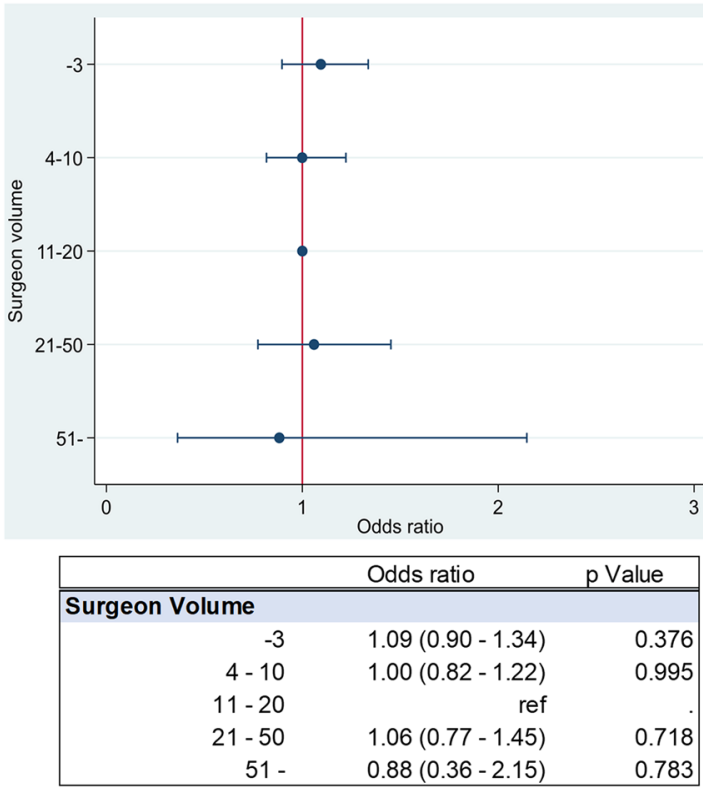

Fig. 1 Forest plot for overall mortality calculated by hierarchical logistic regression models. a Surgeon volume adjusted by risk model variables, b hospital volume adjusted by risk model variables, $\mathbf{c}, \mathbf{d}$ surgeon and hospital volume adjusted by risk model variables includ-

in a dose-dependent manner. This finding suggested that hospital volume can have a crucial impact on postoperative mortality compared with surgeon volume. There are several potential benefits for short-term outcomes that result from a high hospital volume. First, the surgeon can provide referrals to various experts before and after surgery for elderly patients or patients with comorbidities. Second, high-volume hospitals generally have a sufficient cooperative structure

d

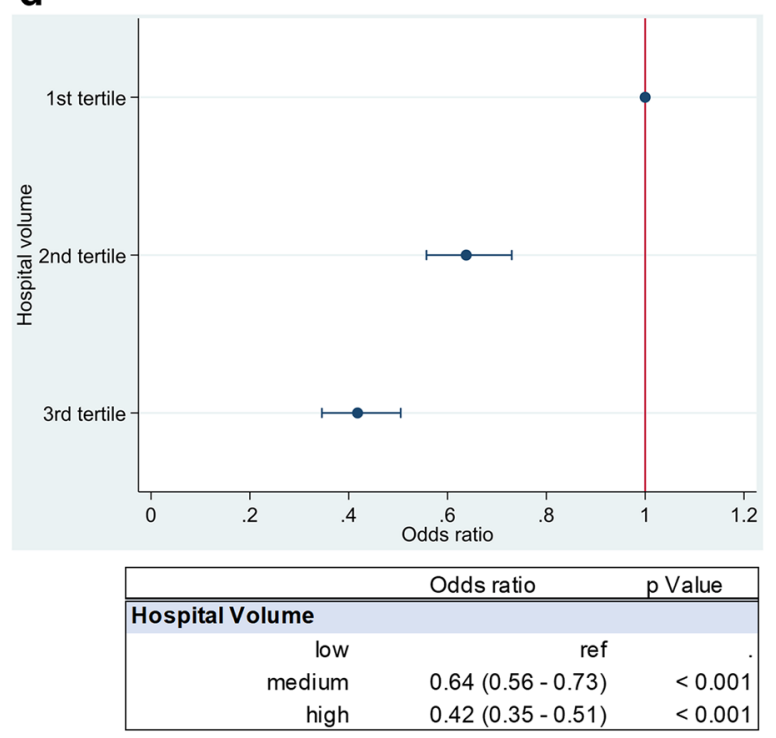

ing hospital volume. Demographic factors, preoperative functional status, pre-existing comorbidities, operative factors and preoperative laboratory data was utilized to adjust for patient-level risk factors

for diagnostic and interventional procedures. Third, surgeon volume will increase in high-volume centers under supervision by experts for gastrectomy, leading to lower mortality compared with surgeons with smaller caseloads. Therefore, centralizing gastrectomy for gastric cancer naturally occurs.

Recently several studies have shown that centralization of gastric cancer surgery is associated with morbidity and mortality rates. In Denmark, Jensen et al. reported that 


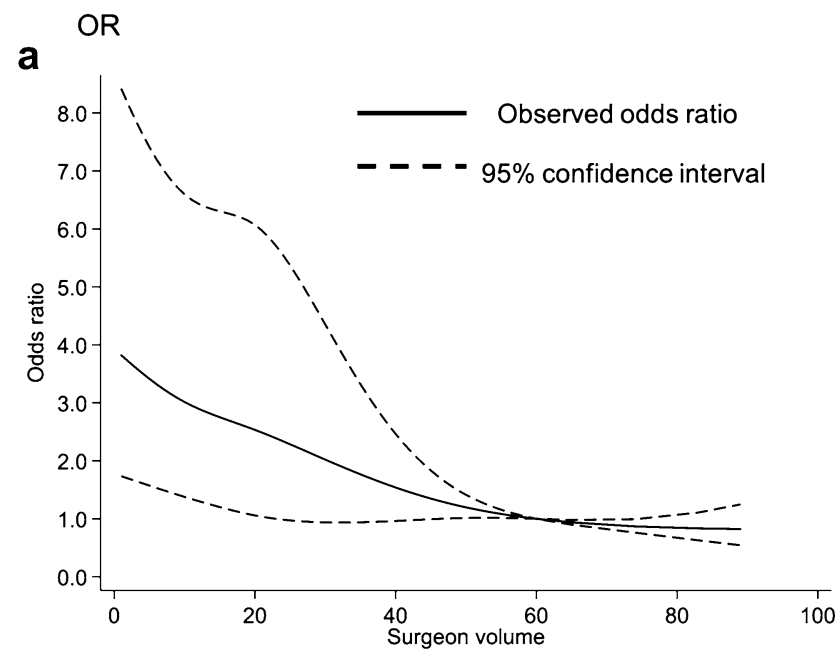

Fig. 2 Odds ratio of mortality after gastrectomy according to surgeon caseload per year calculated by generalized estimating equation logistic regression models with a restricted cubic spline model a adjusted by risk model variables and $\mathbf{b}$ adjusted by risk model variables including hospital volume. Solid lines: observed odds ratio; dashed

centralization of gastric cancer surgery with implementation of national clinical guideline was associated with development in the quality of surgery and lower in-hospital mortality. The 30-days hospital mortality was $2.4 \%$ after centralization (2003-2008) compared to $8.2 \%$ before centralization (1999-2003) [27]. Nelen et al. reported that centralizing gastrectomy improved the number of harvested lymph nodes and successfully introduced laparoscopic gastrectomy [28]. The laparoscopic approach is a complex procedure compared with open surgery, requiring a specialized surgeon and unit. Recent study based on NCD data in Japan showed the incidence of pancreatic fistula was significantly higher in laparoscopic distal gastrectomy compared to open distal gastrectomy [29, 30]. In the current study, the incidence of pancreatic fistula was higher in high-volume hospitals partly because laparoscopic gastrectomy were performed in the high-volume hospitals. On the hand, Lee et al. demonstrated that hospital volume did not directly affect postoperative morbidity and mortality achieved by well-trained beginners of laparoscopic gastrectomy [31]. This finding suggests that surgeon volume is the most crucial factor affecting postoperative outcomes in laparoscopic gastrectomy. Furthermore, it is possible that the patient characteristics depend on each surgeon and hospital. Busweiler et al. reported that elderly patients might benefit specifically from centralization [32]. In our study, low-volume hospitals had significantly older patients and poorer-risk patients with various comorbidities and organ dysfunctions, leading to worse outcomes in low-volume hospital. Although we adjusted for both patient-level and operative factors in this

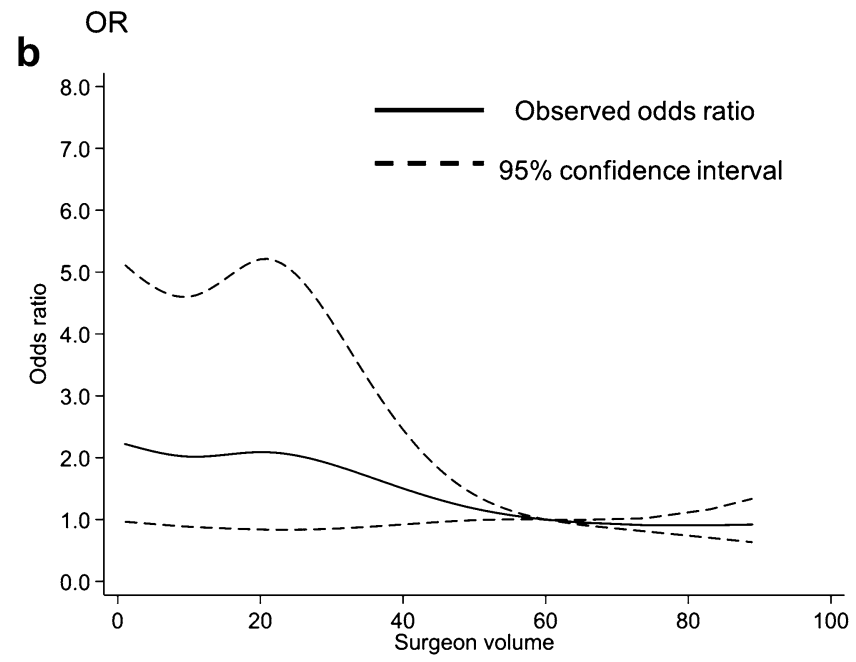

lined: $95 \%$ confidence interval from the logistic regression model. Demographic factors, preoperative functional status, pre-existing comorbidities, operative factors and preoperative laboratory data was utilized to adjust for patient-level risk factors

study, a nationwide population-based study is required to confirm the impact of centralizing gastric cancer surgery on morbidity and mortality.

Our study has some limitations. First, we should consider whether the criteria of surgeon and hospital volume in this study is appropriate. The surgeon volume has been arbitrarily defined according to the data distribution in previous studies. To clarify the criteria of the surgeon volume, we analyzed OR of mortality as shown in Fig. 2, suggesting that the OR of mortality according to surgeon caseload per year reaches the plateau in about 50 cases per year. Also, hospital volume is divided into tertile; low (1-22), medium (23-51) and high (52-404). However, it is possible that this threshold calculated by Japanese population can hardly be expected to apply to clinical practice in Eastern, still less in Western countries. Although this study showed that hospital and surgeon volume are associated with lower postoperative mortality, we should consider the differences of epidemiology, biology and treatment strategy of each country when we determine the concrete threshold of surgeon and hospital volume. Second, our study is limited in that longterm outcomes, such as recurrence-free survival and overall survival, were not evaluated. Most recent report from Netherland demonstrated that centralization of gastric cancer surgery was associated with improved both short and long-term outcome [33]. Interestingly, this report showed that survival improved not only for patients who underwent gastrectomy but also for all patients, irrespective of treatment. It is possible explanation that treating a greater number of patients with gastric cancer in a hospital can lead to improvements throughout treatment management such as preoperative 
diagnosis, perioperative management and chemotherapy in addition to surgical skill. Therefore, further analysis of the impact of surgeon and hospital volume on long-term outcomes after gastrectomy is required in the East, including Japan. Despite these limitations, it is possible that our results have implications for improving healthcare delivery.

\section{Conclusions}

Evaluating hospital volume had a strong impact on postoperative mortality after distal gastrectomy for Japanese patients with gastric cancer in a nationwide web-based data entry system, NCD. Further prospective analysis is required to demonstrate that the centralizing gastric cancer surgery can improve morbidity, mortality and the overall survival of patients with gastric cancer.

Acknowledgements The authors thank all of the data managers and hospitals participating in this NCD project for their great efforts in entering the data analyzed in this study. The authors also thank Prof Hideki Hashimoto and Noboru Motomura, MD, for providing direction for the foundation of the NCD and the working members of the JSGS database committee (Harushi Udagawa, MD; Michiaki Unno, MD; Itaru Endo, MD; Chikara Kunisaki, MD; Akinobu Taketomi, MD; Akira Tangoku, MD; Tadahiko Masaki, MD; Shigeru Maruhashi, MD; Kazuhiro Yoshida, MD). The authors also thank Hiroyuki Konno, MD, for his important suggestions regarding this article. This work was supported in part by a Grant-in-Aid for Scientific Research from the Japan Society for the Promotion of Science, grant number 16K10463 (For M.I.).

Funding None of these organizations had any role in the design and conduct of the study, data collection, data analysis, data management, data interpretation, or the preparation, review, and approval of this manuscript. This work was supported in part by a Grant-in-Aid for Scientific Research from the Japan Society for the Promotion of Science, grant number 16K10463 (For M.I.).

\section{Compliance with ethical standards}

Conflict of interest Hiroaki Miyata and Hiroyuki Yamamoto are affiliated with the Department of Healthcare Quality Assessment at the University of Tokyo. The department is a social collaboration department supported by grants from the National Clinical Database, Johnson \& Johnson K.K., and Nipro Co.

\section{References}

1. Siegel RL, Miller KD, Jemal A. Cancer statistics, 2016. CA Cancer J Clin. 2016;66(1):7-30.

2. Kubota T, Hiki N, Sano T, Nomura S, Nunobe S, Kumagai K, et al. Prognostic significance of complications after curative surgery for gastric cancer. Ann Surg Oncol. 2014;21(3):891-8.

3. Tokunaga M, Tanizawa Y, Bando E, Kawamura T, Terashima M. Poor survival rate in patients with postoperative intra-abdominal infectious complications following curative gastrectomy for gastric cancer. Ann Surg Oncol. 2013;20(5):1575-83.
4. Sierzega M, Kolodziejczyk P, Kulig J, Polish Gastric Cancer Study G. Impact of anastomotic leakage on long-term survival after total gastrectomy for carcinoma of the stomach. Br J Surg. 2010;97(7):1035-42.

5. Ajani JA, D’Amico TA, Almhanna K, Bentrem DJ, Chao J, Das P, et al. Gastric cancer, version 3.2016, NCCN clinical practice guidelines in oncology. J Natl Compr Cancer Netw. 2016;14(10):1286-312.

6. Japanese Gastric Cancer A. Japanese gastric cancer treatment guidelines 2014 (ver. 4). Gastric Cancer. 2017;20(1):1-19.

7. Smyth EC, Verheij M, Allum W, Cunningham D, Cervantes A, Arnold D, et al. Gastric cancer: ESMO clinical practice guidelines for diagnosis, treatment and follow-up. Ann Oncol. 2016;27(suppl 5):v38-49.

8. Dikken JL, van Sandick JW, Allum WH, Johansson J, Jensen LS, Putter H, et al. Differences in outcomes of oesophageal and gastric cancer surgery across Europe. Br J Surg. 2013;100(1):83-94.

9. Birkmeyer JD, Siewers AE, Finlayson EV, Stukel TA, Lucas FL, Batista I, et al. Hospital volume and surgical mortality in the United States. N Engl J Med. 2002;346(15):1128-37.

10. Huo YR, Phan K, Morris DL, Liauw W. Systematic review and a meta-analysis of hospital and surgeon volume/outcome relationships in colorectal cancer surgery. J Gastrointest Oncol. 2017;8(3):534-46.

11. Mahmoudi E, Lu Y, Chang SC, Lin CY, Wang YC, Chang CJ, et al. The associations of hospital volume, surgeon volume, and surgeon experience with complications and 30-day rehospitalization after free tissue transfer: a national population study. Plast Reconstr Surg. 2017;140(2):403-11.

12. Mamidanna R, Ni Z, Anderson O, Spiegelhalter SD, Bottle A, Aylin $\mathrm{P}$, et al. Surgeon volume and cancer esophagectomy, gastrectomy, and pancreatectomy: a population-based study in england. Ann Surg. 2016;263(4):727-32.

13. Mehta A, Efron DT, Canner JK, Dultz L, Xu T, Jones C, et al. Effect of surgeon and hospital volume on emergency general surgery outcomes. J Am Coll Surg. 2017;225(5):666-75. e2.

14. Coupland VH, Lagergren J, Luchtenborg M, Jack RH, Allum W, Holmberg L, et al. Hospital volume, proportion resected and mortality from oesophageal and gastric cancer: a population-based study in England, 2004-2008. Gut. 2013;62(7):961-6.

15. Bachmann MO, Alderson D, Edwards D, Wotton S, Bedford C, Peters TJ, et al. Cohort study in South and West England of the influence of specialization on the management and outcome of patients with oesophageal and gastric cancers. Br J Surg. 2002;89(7):914-22.

16. Miyata H, Gotoh M, Hashimoto H, Motomura N, Murakami A, Tomotaki A, et al. Challenges and prospects of a clinical database linked to the board certification system. Surg Today. 2014;44(11):1991-9.

17. Watanabe M, Miyata H, Gotoh M, Baba H, Kimura W, Tomita N, et al. Total gastrectomy risk model: data from 20,011 Japanese patients in a nationwide internet-based database. Ann Surg. 2014;260(6):1034-9.

18. Kurita N, Miyata H, Gotoh M, Shimada M, Imura S, Kimura $\mathrm{W}$, et al. Risk model for distal gastrectomy when treating gastric cancer on the basis of data from 33,917 Japanese patients collected using a nationwide web-based data entry system. Ann Surg. 2015;262(2):295-303.

19. Gotoh M, Miyata H, Hashimoto H, Wakabayashi G, Konno H, Miyakawa S, et al. National Clinical Database feedback implementation for quality improvement of cancer treatment in Japan: from good to great through transparency. Surg Today. 2016;46(1):38-47.

20. Brisinda G, Crocco A, Tomaiuolo P, Santullo F, Mazzari A, Vanella S. Extended or limited lymph node dissection? A gastric cancer surgical dilemma. Ann Surg. 2012;256(6):e30-1. 
21. Sabesan A, Petrelli NJ, Bennett JJ. Outcomes of gastric cancer resections performed in a high volume community cancer center. Surg Oncol. 2015;24(1):16-20.

22. Jha AK. Back to the Future: Volume as a Quality Metric. JAMA. 2015;314(3):214-5.

23. Hashimoto DA, Bababekov YJ, Mehtsun WT, Stapleton SM, Warshaw AL, Lillemoe KD, et al. Is annual volume enough? The role of experience and specialization on inpatient mortality after hepatectomy. Ann Surg. 2017;266(4):603-9.

24. Birkmeyer JD, Stukel TA, Siewers AE, Goodney PP, Wennberg DE, Lucas FL. Surgeon volume and operative mortality in the United States. N Engl J Med. 2003;349(22):2117-27.

25. Kim CY, Nam BH, Cho GS, Hyung WJ, Kim MC, Lee HJ, et al. Learning curve for gastric cancer surgery based on actual survival. Gastric Cancer. 2016;19(2):631-8.

26. Liang Y, Wu L, Wang X, Ding X, Liang H. The positive impact of surgeon specialization on survival for gastric cancer patients after surgery with curative intent. Gastric Cancer. 2015;18(4):859-67.

27. Jensen LS, Nielsen H, Mortensen PB, Pilegaard HK, Johnsen SP. Enforcing centralization for gastric cancer in Denmark. Eur J Surg Oncol. 2010;36(Suppl 1):50-4.

28. Nelen SD, Heuthorst L, Verhoeven RHA, Polat F, Kruyt PM, Reijnders K, et al. Impact of centralizing gastric cancer surgery on treatment, morbidity, and mortality. J Gastrointest Surg. 2017;21(12):2000-8.

29. Yoshida K, Honda M, Kumamaru H, Kodera Y, Kakeji Y, Hiki $\mathrm{N}$, et al. Surgical outcomes of laparoscopic distal gastrectomy compared to open distal gastrectomy: a retrospective cohort study based on a nationwide registry database in Japan. Ann Gastroenterol Surg. 2018;2(1):55-64.

30. Hiki N, Honda M, Etoh T, Yoshida K, Kodera Y, Kakeji Y, et al. Higher incidence of pancreatic fistula in laparoscopic gastrectomy. Real-world evidence from a nationwide prospective cohort study. Gastric Cancer. 2018;21(1):162-70.

31. Lee HH, Son SY, Lee JH, Kim MG, Hur H, Park DJ. Surgeon's experience overrides the effect of hospital volume for postoperative outcomes of laparoscopic surgery in gastric cancer: multiinstitutional study. Ann Surg Oncol. 2017;24(4):1010-7.

32. Busweiler LAD, Dikken JL, Henneman D, van Berge Henegouwen MI, Ho VKY, Tollenaar R, et al. The influence of a composite hospital volume on outcomes for gastric cancer surgery: a Dutch population-based study. J Surg Oncol. 2017;115(6):738-45.

33. von Putten M, Nelen S, Lemmens V, Stoot J, Hartgrink H, Gisbertz $\mathrm{S}$, et al. Overall survival before and after centralization of gastric cancer surgery in the Netherlands. Br J Surg. 2018. https ://doi.org/10.1002/bjs.10931 